\title{
Retour vers le futur : la reféodalisation du capitalisme moderne ${ }^{1}$
}

\author{
Sighard Neckel*
}

Résumé: Dans de nombreuses sociétés modernes, une transformation sociale est en cours, établissant des privilèges "néo-féodaux" pour les classes fortunées, tandis que les couches inférieures sont confrontées à l'exclusion et au retour du travail forcé. Se référant à un concept analytique développé jadis par Jürgen Habermas, cette transformation est conçue comme une «reféodalisation» du capitalisme moderne. La «reféodalisation» est un mode paradoxal de transformation sociale qui fait apparaître des modèles sociaux pré-modernes comme résultats de la modernisation.

Mots-clés: reféodalisation, sphère publique, inégalité sociale, changement social, capitalisme

\section{Zurück in die Zukunft: die Refeudalisierung des modernen Kapitalismus}

Zusammenfassung: In zahlreichen modernen Gesellschaften findet ein Wandel der sozialen Ungleichheit statt, der neofeudale Privilegien für Vermögende etabliert, während untere Schichten mit Exklusion und der Wiederkehr unfreier Arbeit konfrontiert sind. Mit Bezug auf ein analytisches Konzept, das einst Jürgen Habermas entwickelt hat, wird dieser Wandel als «Refeudalisierung» des modernen Kapitalismus beschrieben. «Refeudalisierung» ist ein paradoxer Modus sozialer Transformation, der als Ergebnis von Modernisierung vormoderne gesellschaftliche Muster entstehen lässt.

Schlüsselwörter: Refeudalisierung, Öffentlichkeit, soziale Ungleichheit, sozialer Wandel, Kapitalismus

\section{Back to the Future: The Refeudalization of Modern Capitalism}

Abstract: In several modern societies a change of social inequality is underway, installing neofeudal privilege for the rich while lower classes experience exclusion and a return of unfree labor. Referring to an analytical concept originally introduced by Jürgen Habermas, this transformation is examined as a "refeudalization" of modern capitalism. "Refeudalization" is a paradoxical mode of social change that leads to pre-modern societal patterns as a result of modernization.

Keywords: refeudalization, public sphere, social inequality, social change, capitalism

* Universität Hamburg, Institut für Soziologie, DE-20146 Hamburg, sighard.neckel@wiso.unihamburg.de.

1 Cet article se base sur une conférence prononcée de la Société suisse de sociologie 2015, le 3 juin 2015 à Université de Lausanne. L'auteur remercie chaleureusement Jacques-Antoine Gauthier, Hermann Kocyba, Olivier Moeschler et Olivier Voirol d'avoir traduit ce texte à partir de la version originale allemande. 
Dans son importante étude sur la "crise de l'espace public» en 2011, notre collègue Kurt Imhof - qui vient de décéder prématurément - a donné une image saisissante de la déliquescence progressive de l'espace public, et notamment de la communication médiatique à l'ère du néolibéralisme. Kurt Imhof a montré que, depuis les années 1980, les médias se sont toujours plus détachés des contextes sociopolitiques, religieux et sociaux dans lesquels ils étaient insérés, ce qui a provoqué un processus intense de concentration économique du paysage médiatique (cf. Imhof 2011, 85 ss.). Depuis, les entreprises médiatiques recherchent avant tout le succès commercial d'une communication banalisante adressée à un public de plus en plus nivelé. Une spirale négative de prolifération de "contenus médiatiques de faible qualité" s'est mise en place, poussée par le rôle prépondérant des portails en ligne et par la "simplicité croissante» d'une "gestion médiatique commerciale de l'indignation" (cf. Imhof 2011, 147 ss.).

La fonction publique des médias consistant à thématiser, entre la société et l'Etat, les problèmes qui peuvent concerner tous les citoyens, a été remplacée par un populisme médiatique dans lequel le moment épisodique, particulier, moralisateur, émotionnel et facilement consommable s'est progressivement substitué aux standards journalistiques de l'enquête, de l'information et du débat. Toujours selon Imhof, au citoyen a succédé un consommateur médiatique qui, au travers de la like-economy des réseaux sociaux et d'une communication Internet exempte de toute profondeur réflexive, participe lui-même à sa propre élimination en tant que citoyen. Ceci se ferait aux dépens des apports politiques des médias, dont dépendent pourtant les démocraties. Ainsi, les médias ne seraient plus capables, ni de remplir la fonction de communiquer publiquement des énoncés sur de nouveaux problèmes, ni d'exercer un contrôle efficace sur l'autoritarisme étatique, ni de servir à l'intégration de perceptions sociales différentes (cf. Imhof 2014, 314).

Les phénomènes de crise du secteur des médias diagnostiqués par Kurt Imhof ont des conséquences d'autant plus fatales que la perte de sens de la communication publique, sous le coup du néolibéralisme et de l'économie globale sur la sphère publique, étend la pression de la marchandisation jusque sur le quotidien médiatique de la population. Les médias deviennent ainsi le pilier d'une post-démocratie qui voit s'évaporer la chose publique de l'espace médiatique à mesure que les décisions politiques se déplacent effectivement des instances légitimées politiquement à des cercles d'intérêts privés, économiquement puissants, et qui parviennent à s'imposer (cf. Crouch 2004). Et c'est exactement cette privatisation de la communication médiatique amenée par l'économie qui permet de parler d'un «nouveau changement structurel de l'espace public», selon l'analyse de Kurt Imhof.

Avec ce constat d'un nouveau "changement structurel de l'espace public», Kurt Imhof s'est explicitement référé à une importante étude qui l'a très profondément 
marqué durant toute son existence de sociologue et d'intellectuel. On veut bien évidemment parler du texte de Jürgen Habermas (1978) L'espace public: archéologie de la publicité comme dimension constitutive de la société bourgeoise. Paru en 1962, ce livre aboutissait à des conclusions similaires à celles auxquelles parvient Kurt Imhof 50 ans après, même si les contours du paysage médiatique des années 1960 étaient encore très différents. Mais Habermas avait mis au centre de son analyse exactement ce processus de "privatisation " de la sphère publique qui constitue chez Kurt Imhof le noyau de son diagnostic d'une crise de la publicité médiatique.

A l'instar de Imhof, Habermas entend par "espace public » une sphère qui sert de médiateur entre la société et l'Etat et qui rend possible une participation équitable de toutes les citoyennes et tous les citoyens au débat public sur des affaires générales et sur le pouvoir politique. Ce faisant, l'autorité étatique est un adversaire de l'espace public et non pas une partie de celui-ci, comme c'est encore le cas dans le système féodal - dans lequel "public» désigne la représentation visible du pouvoir -, où les pouvoirs publics sont aussi peu séparés de la Cour que le budget étatique l'est du domaine du souverain.

Si la sphère publique naît à l'origine comme instance de limitation du pouvoir personnifié dans le système de domination monarchique, au $19^{\text {ème }}$ siècle déjà apparaît selon Habermas, avec la presse d'affaires, l'utilisation de l'espace public pour des intérêts privés par calcul économique (cf. Habermas 1978, 189 ss. et 231 ss.). Le public devient alors une composante d'une culture commerciale de la consommation, et la représentation du pouvoir personnifié fait son retour en tant que relations publiques de personnes privées, puissantes économiquement et qui font passer leurs intérêts particuliers pour l'intérêt général. Emerge alors une forme moderne de représentation du pouvoir, que Habermas $(1973,68)$ nomme «reféodalisation de l'espace public» parce qu'elle signifie un retour, dans le cadre de la société bourgeoise, à la privatisation de l'espace public et rappelle l'époque pré-bourgeoise durant laquelle il n'y avait pas encore de séparation entre affaires publiques et intérêts privés.

Avec Habermas, nous pouvons donc parler de "reféodalisation " dès lors que, dans le paysage médiatique, le pouvoir économique de quelques-uns se traduit directement par la domination exercée sur l'espace public et que les intérêts privés déterminent les modalités de négociation des affaires publiques. Par là, il y a un brouillage de la distinction entre l'Etat et la société, et la sphère publique retombe dans un état de représentation du pouvoir qui était typique de l'ordre social prémoderne.

Les diagnostics de Jürgen Habermas et de Kurt Imhof concordent en cela qu'ils identifient la prédominance d'intérêts économiques comme étant les principales forces qui ébranlent la légitimité de la sphère publique dans la démocratie. Chez Habermas, c'est la formation de monopoles économiques qui fait à nouveau pénétrer dans l'espace public des formes de pouvoir prébourgeois. Kurt Imhof a lui-même ajouté à cela - dans le sens d'un diagnostic du présent - le changement des médias 
en ligne poussant à la privatisation : ceux-ci créent dans le réseau Internet de nouveaux "paysages d'attention " (Imhof 2014, 327) situés au-delà de tout débat public sur des affaires générales. Dans son étude sur ce récent changement structurel, Kurt Imhof n'a pas parlé de "reféodalisation». Peut-être le mot lui semblait-il quelque peu décalé par rapport à la modernité de la communication en ligne. Néanmoins, Google, Apple, Facebook, Microsoft et d'autres régents de l'ère du big data sont bien des exemples flagrants montrant qu'il y a belle lurette qu'au réseau d'Internet originellement distribué se sont substitués des empires privés du pouvoir médiatique digital. A l'échelle mondiale, Facebook est utilisé par quelque 1.4 milliards d'humains, mais contrôlé par une seule personne (Lanier 2015).

\section{La reféodalisation comme concept de l'analyse sociologique}

Mais ce n'est pas que du point de vue de la théorie des médias que l'état actuel de l'espace public doit susciter notre intérêt. Les transformations de la sphère publique et des formes médiatiques qu'elle adopte constituent pour l'analyse sociologique un symptôme général du changement sociétal. Habermas lui-même, en revenant sur son étude de 1962, définit l'état de l'espace public comme un «indicateur politique»: nulle part ailleurs pouvait-on identifier plus tôt les problèmes des démocraties actuelles et le déclin des structures sociétales modernes qu'en se fondant sur l'état de la sphère publique médiatique (cf. Habermas 2015). Les crises actuelles de l'espace public sont donc aussi à considérer comme un indicateur des problèmes de l'évolution de la société dans son ensemble.

La thèse centrale de ma présentation consiste à dire que ce n'est pas seulement parce qu'on parle à nouveau aujourd'hui, dans les sciences sociales, d'un «changement de structure de l'espace public» empli de crises, que la notion habermassienne de «reféodalisation» acquiert une nouvelle actualité. Bien plus, je souhaite montrer qu'un changement est actuellement en cours dans de nombreux secteurs de la société, qui voit émerger à nouveau des hiérarchies et des structures de pouvoir prémodernes, dans le sillage d'une modernisation néolibérale de l'économie et de la société. Ceci non pas comme un retour aux temps anciens, mais comme un résultat paradoxal de transformations sociales qui recréent de l'ancien en tant que nouveau et qui, ce faisant, produisent des modes «néo-féodaux» de répartition de la richesse, de la reconnaissance et du pouvoir (cf. Neckel 2010; Neckel 2013; Neckel 2014; Neckel 2015).

Dans le modèle analytique de la «reféodalisation", les horizons temporels sociologiques s'entremêlent: le nouveau n'est pas simplement la conséquence de processus linéaires de modernisation, mais est créé au cours d'un changement social qui fait émerger de l'ancien comme du nouveau dans la mesure où des formes d'organisation actuelles de l'économie et de la société réactualisent, sous une forme très 
moderne, des motifs traditionnels de l'ordre social. Les études de Habermas sur le changement de structure de l'espace public constituent le cas d'étude exemplaire de ce modèle d'analyse, puisqu'il décrit l'espace public médiatique comme un marché des opinions qui fait finalement renaître sous forme commerciale la vieille structure féodale du public comme simple représentation d'un pouvoir personnifié.

Du point de vue du changement social, la reféodalisation matérialise ainsi un processus beaucoup plus complexe qu'un retour vers le passé. Bien plus, l'analyse exemplaire de la reféodalisation de l'espace public par Habermas signale que le changement de structure en question est le résultat d'un processus de modernisation paradoxal, dans le cadre duquel se ré-établissent des structures sociales qui ont leur origine dans des époques prébourgeoises de l'histoire de la société.

Il est vrai que dans la théorie sociale dominaient jusqu'à aujourd'hui des modèles de changement social qui opposaient aux hypothèses d'un changement "progressif» - comme dans la théorie de la modernisation - la preuve de processus sociaux de déclin "régressifs", comme par exemple dans la première Théorie critique de l'Ecole de Francfort. A l'inverse, je voudrais montrer que la dichotomie de formes d'évolution "progressives» et « régressives» du capitalisme doit être complétée par des modèles plus complexes de changements sociaux paradoxaux. Dans ce contexte, je considère la reféodalisation comme un exemple de changement social qui concerne une modernisation paradoxale au cœur du capitalisme moderne. Il ne mène pas droit au passé, mais désigne une dynamique sociale contemporaine qui accomplit la modernisation comme une renonciation aux maximes de l'ordre social bourgeois.

\section{L'« effet Vertigo » : contre-mouvements paradoxaux du changement social}

Dans le mode de la reféodalisation, la modernisation capitaliste s'opère ainsi comme un processus intrinsèquement à contresens - comme une roue dentée qui roule à l'envers et qui fait avancer son objet à reculons. Au cinéma, on appelle ce mouvement inversé un dolly zoom, ou aussi l'"effet Vertigo", depuis que cet effet a été utilisé pour la première fois dans le film d'Alfred Hitchcock Vertigo en 1958 (cf. Neckel 2013, 47-48). L'« effet Vertigo» se produit lorsque la caméra s'approche d'un objet tout en faisant un zoom arrière - sans changer le cadrage. Il en résulte un effet visuel d'aspiration utilisé par Hitchcock pour exprimer filmiquement le vertige de son acteur principal, James Stewart.

C'est à cet "effet Vertigo" du changement social auquel je vais m'intéresser dans ce qui suit. A cette fin, j'introduis ici le terme de "reféodalisation» en tant que concept sociologique clé pour la compréhension de la dynamique du développement actuel des sociétés capitalistes modernes. J'aimerais montrer qu'en tant que modèle paradoxal du développement social, la perspective analytique d'une «reféodalisation " de la modernité capitaliste permet d'éclairer l'évolution de la société en général, qu'il 
s'agisse de l'assèchement de la sphère publique à travers la privatisation du pouvoir des médias ou de processus de transformation inverses dans d'autres domaines fonctionels du capitalisme actuel.

Par conséquent, je distingue quatre dimensions des processus de reféodalisation dans l'ordre social actuel. Premièrement, par rapport à la structure sociale et à une transformation des inégalités sociales qui révèlent des signes évidents de féodalisation dans leur manière de favoriser le renforcement du corporatisme et la polarisation de situations sociales extrêmement différentes. Deuxièmement, au sujet de l'organisation des processus économiques et du statut des groupes dominants sur les marchés économiques, qui n'agissent pas en entrepreneurs, mais en rentiers. Troisièmement, sur le plan normatif, en tant que reféodalisation des valeurs en lien avec l'ordre de légitimation du marché de la finance capitaliste. Cela concerne essentiellement le remplacement du principe du rendement (Leistung) par les préceptes du pur succès financier, ainsi que la reféodalisation de sa reconnaissance, qui se réalise au travers des célébrités de l'ère médiatique et le mode d'exposition des people. Quatrièmement, enfin, en raison de la reféodalisation de l'Etat social, les politiques sociales publiques sont reprivatisées sous la forme de fondations et de donations et les droits sociaux se transforment en étant soumis à cette charité privée (cf. Neckel 2013, 49 ss.).

\section{$4 \quad$ Les inégalités sociales par le bas: le retour du travail forcé}

Pour des raisons de temps, dans le cadre de cette conférence, j'aimerais me limiter à la première dimension de la structure sociale et juste aborder la deuxième dimension, celle des groupes dominants actuels de l'économie. Si l'on parle de la structure sociale, on constate qu'au cours des vingt dernières années, diverses formes de reféodalisation ont émergé dans de nombreux pays capitalistes. Ce développement se traduit par des avantages importants accordés sans contreparties aux élites de la société, qui bénéficient d'une opportunité d'enrichissement unique dans l'histoire, alors que les couches inférieures ne se sont pas seulement paupérisées, mais aussi de plus en plus exposées à des conditions de travail qui ne correspondent plus aux standards modernes élémentaires en matière de relations contractuelles.

Le fait que les formes d'organisation actuelles du travail se dissocient souvent à l'échelle globale des normes d'égalité en matière de droit représente, par exemple dans le domaine du travail domestique, un phénomène transnational de masse. Ainsi, dans la recherche sur les mutations globales d'une force de travail en général féminine engagée dans les métropoles comme personnel de maison, un débat s'est ouvert sur la question de savoir si l'on ne pouvait pas voir dans le travail domestique des migrants un retour à l'ancienne catégorie des domestiques et une reféodalisation des rapports de travail domestiques (cf. Ehrenreich et Hochschild 2003; Lutz 2008). Les nouvelles femmes de ménage qui sont à la recherche d'un emploi sur les marchés globaux de 
l'emploi domestique, se trouvent par conséquent prises entre différentes situations de dépendance personnelle, qui révèlent la séparation bourgeoise et moderne du privé et du public, du lieu de travail et du domicile, du paiement d'un salaire et du don de soi. Ainsi se développent des rapports de travail et d'exploitation dans lesquels aucune égalité contractuelle ne peut s'établir et qui favorisent la violence et la contrainte sexuelles. Les protections sociales dont bénéficie cette main d'œuvre sont minimales. De plus, lorsque ces femmes ne se trouvent pas de manière illégale dans les pays d'accueil, leurs autorisations de séjour sont considérablement limitées. Ainsi leur travail s'accomplit-il souvent de manière invisible dans la sphère privée de leur employeur, dans les ménages desquels ces femmes vivent en étant privées de toute liberté de mouvement. En particulier dans le gigantesque segment professionnel à l'échelle mondiale des global care chains, dans lesquels des femmes abandonnent leur pays d'origine plus pauvre pour effectuer des travaux de soin et de ménage dans les pays plus riches, les rapports de travail se sont aussi transformés en rapports de dépendance personnelle, ce qui constitue un retour en arrière par rapport à tous les progrès de la société moderne au niveau de la reconnaissance des droits et renvoie les femmes concernées à des rapports de domination néo-féodale.

Nous connaissons de nombreuses formes extrêmes de travail forcé au sein de la foule immense des travailleurs industriels itinérants, en particulier en Asie, en Afrique et dans certains pays d'Amérique du Sud. Ce sont eux qui érigent les nouvelles mégapoles en Chine, qui travaillent sans permis dans les chantiers des Emirats arabes, qui sont assignés au travail obligatoire organisé par l'Etat dans les champs de coton ouzbèkes au profit des oligarques, ou qui comptent parmi les quelque vingt millions d'individus qui, en Inde, vivent dans une situation de servitude de dettes (cf. Bales 2012). Certes, ces formes actuelles d'esclavagisme sont liées à d'anciennes structures de domination racistes, comme par exemple l'esclavagisme ethnique dans des pays africains tels que le Soudan ou la Mauritanie (cf. Bales et al. 2009, 91 ss.). Pourtant les experts des organisations des droits humains comme Walk Free Foundation (2014) et de l'Organisation internationale du travail (OIT) sont unanimes à reconnaître que la période moderne de la globalisation est associée à un renouveau de l'esclavagisme et que celui-ci y prend une ampleur inconnue jusqu'ici. Alors que des résultats de recherches sociologiques estiment qu'entre 30 et 35 millions d'individus (parmi lesquels environ un million en Europe) vivent aujourd'hui dans des conditions de violence et de privation de droits proches de l'esclavage (cf. Bales et Soodalter 2009; Bales 2012; Walk Free Foundation 2014), l'OIT estime en 2014 qu'à l'échelle de la planète, 21 millions de personnes sont victimes du travail forcé, et présume que le nombre réel des personnes dans cette situation est en fait bien plus élevé; c'est grâce à elles qu'on réalise un profit d'environ 150 milliards de dollars par an (cf. Datta et Bales 2013b; OIT 2014).

Au XXI ${ }^{\text {ème }}$ siècle, on compte sur terre davantage de travailleurs forcés qu'il n'y en a jamais eu de toute l'histoire de l'humanité (Bales et al. 2009, VII). A côté de la 
globalisation, la croissance de la population a contribué à créer une situation historique unique d'abaissement du coût du travail forcé. En outre, les flux de migration planétaire sont exploités économiquement. Les migrations étant communément faites de manière illégale, elles laissent le plus souvent les migrants sans protection. La réduction des forces de travail à de simples matériaux et l'exploitation impitoyable du capital corporel ne sont de ce fait guère inconnues dans les centres de la mondialisation. Le travail forcé et le trafic d'êtres humains, formes extrêmes de l'exploitation, mais aussi les formes modernes de servitude de dettes se rencontrent également dans les pays occidentaux (cf. Bales et Soodalter 2009; Datta et Bales 2013a; Datta et Bales 2014). L'industrie de la viande, les mines de charbon, la construction, les soins domestiques, les tâches élémentaires ou le secteur agricole sont sous l'égide d'entreprises occidentales et de formes sous-entrepreneuriales d'absence de droits et de contrôle personnel des relations de travail, en rupture avec toutes les normes modernes de liberté juridique en matière de participation au marché du travail.

On observe - en particulier dans le domaine en expansion planétaire des tâches élémentaires - une déqualification du prolétariat de service que l'on connaissait jusqu’à présent. Celui-ci tend à devenir le «dépotoir » de la société du travail moderne, dans lequel les salaires ne permettent plus d'assurer la propre subsistance, dans lequel des rapports de dépendance personnelle et non plus contractuels sont établis et dans lequel ceux qui y travaillent sont privés de toute forme de sécurité sociale, de qualification et d'ascension professionnelles, qui constituent par ailleurs la base des rapports de travail modernes. Au lieu de cela s'exerce ce que la recherche spécialisée appelle une «fatalité de statut lié au marché» (Bahl 2014), pour décrire l'absence complète de perspectives de ces travailleuses et travailleurs précarisés à l'extrême. D'un point de vue historique, le fatalisme à l'égard d'une situation sociale apparemment immuable est la caractéristique typique d'un ordre social marqué par la pauvreté de statut. Lorsque ce fatalisme de statut apparaît dans la société de marché moderne, il révèle une reféodalisation des conditions de travail. Ce sont les tendances modernes d'une marchandisation sans frontières du travail qui représentent au final un retour à des formes de catégories sociales de bas statut telles que nous les connaissions dans la période pré-industrielle.

\section{Les inégalités sociales «par le haut» : les nouvelles oligarchies}

Si le retour du travail forcé et d'une précarité forte et permanente représentent les formes les plus négatives d'une reféodalisation de la partie la plus basse de la hiérarchie sociale, la reféodalisation des couches économiques supérieures s'accomplit d'une manière qui n'est pas moins en rupture avec les principes bourgeois que celle du travail se réalisant au-delà de l'égalité contractuelle et de la garantie d'une existence sociale. 
Une nouvelle oligarchie de richesse s'est développée au sommet de la structure sociale, à la faveur d'un accroissement de patrimoine unique dans l'histoire. Cette opulence sans précédent ne s'explique pas par des principes économiques modernes comme la performance ou le mérite, la concurrence ou les succès commerciaux, comme cela serait le cas dans un ordre social capitaliste et bourgeois, mais par des stratégies de protection des privilèges dont l'origine remonte à l'ère pré-capitaliste. Prenons l'exemple de l'augmentation des revenus les plus élevés au sein de l'élite du management économique. Aux Etats-Unis, au milieu des années 1960, le rapport entre les revenus des PDG et les salaires moyens était de vingt contre un. En 2012, dans ce même pays, ce rapport des salaires des PDG (CEO Pay Ratio) au salaire moyen des employés s'élevait à 273 (Mishel 2013). Il en va de même dans les sociétés européennes qui considèrent toujours qu'elles reposent sur des «économies sociales de marché». Ainsi, en 1989, les directeurs des trente plus grosses entreprises cotées en bourse en Allemagne recevaient un salaire annuel de 500000 DM, ce qui correspondait à l'époque effectivement à vingt fois le salaire moyen des employés. En 2010, les revenus annuels des directeurs s'élevaient à six millions d'euros, un montant deux cents fois supérieur à celui des salaires moyens des employés (cf. Wehler 2013, 79).

Si l'on cherche à expliquer les raisons de ces gains gigantesques, on constate que pas un seul facteur économique ne permet de mettre en relation cette explosion des revenus du top-management avec une augmentation significative des performances de leurs représentants, qui aurait conduit à un accroissement soudain de leur productivité. Les revenus les plus élevés ne correspondent pas à la performance (pay for performance) mais sont bien l'expression de l'accroissement du pouvoir au sein des directions et des conseils d'administration des sociétés anonymes, ce qui permet de soustraire davantage de revenus aux entreprises, plutôt que le résultat d'une contribution exceptionnelle au rendement de ces dernières.

Cette forme de maximisation du profit repose économiquement sur le contournement des processus du marché. En effet, une activité sur le marché est toujours liée à l'incertitude quant à savoir si son engagement sera bel et bien récompensé. Bien qu'elle soit une précondition constitutive des marchés, la concurrence est en mesure de déjouer les perspectives de bénéfice, parce qu'elle peut anéantir les recettes attendues. Par conséquent, l'option la plus avantageuse est toujours de s'assurer des revenus sans être inquiété par la concurrence (cf. Neckel 2015). Les possibilités de développer un tel rapport à ses propres ressources, aux antipodes du marché, lesquelles sont cependant distribuées de manière extrêmement inégalitaire. Les classes supérieures de la société disposent de nombreuses possibilités d'obtenir les plus hauts revenus, sans prestations ni concurrence. Les positions statutaires des groupes favorisés dans des directions, des conseils d'administration et autres organes de gestion économique leur donnent l'occasion de s'assurer différents privilèges, sous forme de rémunérations directes, de réductions, de primes garanties ou de droits à la pension. 
Sur le modèle de «l'effet Matthieu» (Merton 1968), un système d'attribution d'avantages cumulatifs émerge, ne renvoyant pas à des acteurs individuels, contrairement à l'analyse classique de Merton, mais au groupe fermé des dirigeants économiques (cf. DiPrete et Eirich 2006). Apparaît dans son cadre une sorte de "marché du statut" (Aspers 2007, 435 ss.) dans lequel des privilèges sont négociés. Les marchés du statut se caractérisent par le fait que le classement des valeurs n'est pas déterminé par des standards comme la "qualité», par exemple, mais par les structures sociales ayant produit les acteurs du marché.

Ce qu'un acteur gagne ou donne sur un marché du statut dépend par conséquent de la position qu'il occupe dans un ordre statutaire. Lorsque les acteurs bénéficiant d'un statut élevé communiquent entre eux, en se reconnaissant les uns les autres à leurs positions financières au sommet, le bien financier qu'ils négocient prend de la valeur indépendamment de tout critère de performance. Et, sur un marché du statut, ce n'est pas à de tels critères de performance que les acteurs font attention mais à la position sociale qui peut être acquise, renforcée ou perdue au moyen des avantages acquis.

Ce n'est pas la demande sur le marché qui est décisive sur les marchés du statut des couches dirigeant l'économie mais la position sociale des groupes favorisés, qui leur permet de s'assurer réciproquement des avantages au détriment de l'entreprise et de l'environnement économique. Ces stratégies sont rendues possibles par la structure sociale d'un groupe fermé, dont les membres témoignent de grandes similarités et disposent de positions hiérarchiques au sein de directions et de conseils d'administration, qui leur permettent de transformer la direction d'entreprise en structures d'opportunités au service de leurs propres privilèges.

Les règles de l'action d'un tel exercice du pouvoir économique sont généralement décrites, dans l'ensemble, comme des stratégies d'extraction de rentes (cf. Stiglitz 2012, 28 ss.), puisqu'il s'agit d'extraire aux entreprises des revenus élevés sans contreparties, en s'assurant ainsi des rentes. Ici, les bénéficiaires se voient attribuer un droit particulier restreignant la concurrence, jusqu'à la remplacer complètement, ce qui conduit à une distribution des revenus basée sur le principe de gain selon lequel «le gagnant rafle tout» (the winner takes it all).

Le recours à de telles rentes, extraites de l'entreprise à titre d'avantages privilégiés, transforme aussi toute la structure des inégalités sociales en raison de cette extraordinaire croissance de richesses à son sommet. Le chercheur américain spécialiste de la structure sociale David Grusky de l'Université de Stanford voit dans les manières très différenciées de disposer des rentes entre les classes sociales la principale raison de l'accroissement considérable des inégalités sociales au cours des trente dernières années (cf. Grusky et Weeden 2011). Alors que dans les couches inférieures se pratique une politique de destruction de la rente, à l'image de l'abaissement du salaire minimum, dans les couches supérieures en revanche le management d'élite bénéficie du succès durable des stratégies de création de rente, en particulier à titre de garanties 
de revenus exorbitants. Tout comme les garanties de prestations pour les bas salaires furent détruites avec le néolibéralisme, elles furent massivement recréées au bel étage de la hiérarchie sociale. Concernant des sociétés européennes comme l'Allemagne ou la Suisse, on peut aussi décrire ce développement comme une «marchandisation asymétrique» qui touche les différentes classes sociales de manière très différente. Alors que les couches inférieures sont livrées à des marchés du travail déréglementés, allant jusqu'au retour du travail forcé, une série d'arrangements garantissant des revenus élevés furent créés pour les couches favorisées, éliminant ainsi les processus du marché et de la concurrence (cf. Neckel 2015).

Le privilège des rentes sans activité, dont les montants spectaculaires resultent de l'exploitation de l'environnement économique et de structures d'opportunités, n'est pas le seul facteur conduisant aujourd'hui à une forme de capitalisme féodal et à une "nouvelle âge d'or" (new gilded age), comme la décrit actuellement la recherche internationale sur les inégalités (cf. Krugman 2014). D’autres facteurs de reféodalisation qui ne relèvent pas moins de l'arsenal historique des privilèges féodaux sont directement de nature politique. Le traitement préférentiel inouï accordé à une classe opulente globalisée dont les revenus gigantesques proviennent avant tout des marchés financiers est le résultat d'une sorte d'économie du don étatique en faveur des possédants (cf. Stiglitz 2012, 47 ss.). Dans la plupart des pays occidentaux, des cadeaux à hauteur de milliards ont été faits au cours des vingt dernières années sous forme de réductions massives d'impôts sur les revenus du capital, sur les héritages et les hauts salaires. La politique néolibérale de réduction des impôts pour les riches, pour les entreprises et les banques qui sont sous leur contrôle, est due principalement au fait que les groupes sociaux fortunés sont parvenus, sur le plan politique, en raison de leur pouvoir économique, à disposer d'un droit de veto effectif sur toute entrave à leurs intérêts économiques.

\section{La reféodalisation dans la société contemporaine}

Ainsi, ce ne sont pas les principes bourgeois et capitalistes de la performance et de la concurrence qui décident du revenu et de la richesse mais le statut social, le pouvoir économique et les faveurs du pouvoir politique. Ces évolutions conduisent toutes à ce que les sociétés modernes contemporaines témoignent d'une inégalité sociale atteignant les taux du début du $19^{\mathrm{e}}$ siècle, comme l'ont récemment montré les travaux de Thomas Piketty. A cette époque, le décile le plus fortuné de la population disposait de plus de $80-90 \%$ de la richesse globale alors qu'aujourd'hui, dans des sociétés comme l'Allemagne, ce taux est revenu à environ $70-75 \%$, après que le capitalisme d'après-guerre régulé par l'Etat social eut fait chuter ce taux à 45-55\% (cf. Piketty 2013, 541 ss., 591 ss.). Le coefficient de Gini d'un pays comme l'Allemagne est d'ores et déjà de 0.78 en 2012, et il n'est dépassé que par les Etats-Unis, 
parmi les pays souffrant la comparaison, dont le taux est de 0.87 (cf. Grabka et Westermeier 2014, 156).

Dans ce contexte, Thomas Piketty parle du retour d'un «capitalisme patrimonial» basé sur l'hyper-concentration du capital entre les mains d'une nouvelle classe oligarchique fortunée (cf. Piketty 2013, 541 ss.). La société patrimoniale d'aujourd'hui contribue au développement de structures dynastiques de pouvoir économique dans lesquelles l'héritage et le mariage jouent à nouveau un rôle essentiel, et où mettre la main sur un patrimoine élevé apporte une aisance que les études, le travail et la performance ne peuvent permettre d'atteindre (cf. Piketty 2013, 546).

Au regard d'une perspective historique à long terme, un des paradoxes de ce changement social veut qu'aujourd'hui le segment le plus riche d'une bourgeoisie économique ayant acquis sa prospérité et son influence grâce au marché et à la concurrence à l'époque capitaliste cherche, telle l'aristocratie d'autrefois, à renforcer sa propre position par les mêmes moyens d'extraction de la rente et du pouvoir dynastique hérité. Ayant ce point commun historiquement, les gros propriétaires de capitaux forment avec les riches investisseurs sur les marchés financiers et l'élite du management d'entreprise une classe fermée et corporative. Sa position dominante dans les processus économiques de la mondialisation et du capitalisme financier est tributaire d'une reféodalisation du capitalisme moderne et donc du paradoxe d'un développement social à rebours. Cette position économique néo-féodale est liée à la croissance de son pouvoir politique dans les post-démocraties actuelles - un pouvoir politique que les classes supérieures utilisent en particulier pour défendre leurs intérêts économiques. Et cette union du pouvoir économique et du pouvoir politique entre les mains de la nouvelle oligarchie opulente rappelle une sorte de formation de classe prémoderne dans laquelle une séparation des sphères de la politique et du marché, du droit et de l'économie n'avaient pas encore cours.

C'est le mérite de l'étude de Jürgen Habermas consacrée à la «transformation structurelle de l'espace public ", qu'il menait jadis à partir de l'exemple de la reféodalisation de l'espace public, que de montrer de quelle manière le capitalisme peut dissiper, au cours de son processus de modernisation, ce caractère bourgeois dans lequel il puisait son origine, perdant ainsi également ses propriétés normatives qui en ont fait une nouveauté historique. Cela concerne, d'un côté, cette sphère que Habermas considérait lui-même il y a plus de 50 ans: le processus contradictoire de formation publique de la volonté et des intérêts privés, ayant pour conséquence la re-transformation des formes bourgeoises antérieures de l'échange public en une représentation de la domination des intérêts privés. C'est là que l'on retrouve la discussion sur la nouvelle crise de l'espace public que Kurt Imhof a initiée. Comprise comme le paradigme d'une transformation sociale saisie comme un tout, la théorie de la reféodalisation du capitalisme moderne met aussi au jour d'autres sphères importantes de la société actuelle. C'est ainsi qu'en abordant ces formes drastiques de changement social à l'aide d'une théorie de la reféodalisation, on comprend mieux 
la paupérisation actuelle du travail, la résurgence de formes extrêmes d'exploitation, tout comme l'apparition de nouvelles oligarchies opulentes ainsi que la croissance d'un pouvoir dynastique.

\section{Références bibliographiques}

Aspers, Patrik. 2007. Wissen und Bewertung auf Märkten. Berliner Journal für Soziologie 17: 431-449. Bahl, Friederike. 2014. Lebensmodelle in der Dienstleistungsgesellschaft. Hamburg: Hamburger Edition.

Bales, Kevin. 2012. Disposable People: New Slavery in the Global Economy. 3rd revised edition. Berkeley: University of California Press.

Bales, Kevin et Ron Soodalter. 2009. The Slave Next Door. Human Trafficking and Slavery in America Today. Berkeley: University of California Press.

Bales, Kevin, Zoe Trodd et Alex Kent Williamson. 2009. Modern Slavery: The Secret World of 27 Million People. Oxford: Oneworld.

Crouch, Colin. 2004. Post-democracy, Cambridge: Polity Press.

Datta, Monti Narayan et Kevin Bales. 2013a. Slavery in Europe. Part 1: estimating the dark figure. Human Rights Quarterly 35: 817-829.

Datta, Monti Narayan et Kevin Bales. 2013b. Slavery is bad for business: analyzing the impact of slavery on national economies. Brown Journal of World Affairs 19: 205-223.

Datta, Monti Narayan et Kevin Bales. 2014. Slavery in Europe. Part 2: testing a predictive model. Human Rights Quarterly 36: 277-295.

DiPrete, Thomas A. et Gregory M. Eirich. 2006. Cumulative advantage as a mechanism for inequality: a review of theoretical and empirical developments. Annual Review of Sociology 32: 271-297.

Ehrenreich, Barbara et Arlie Hochschild (dir.) 2003. Global Women. Nannies, Maids, and Sex Workers in the New Economy. New York: Metropolitan.

Grabka, Markus M. et Christian Westermeier. 2014. Anhaltend hohe Vermögensungleichheit in Deutschland. DIW Wochenbericht 9: 151-164.

Grusky, David B. et Kim A. Weeden. 2011. Is market failure behind the takeoff in inequality? Pp. 90-97 in The Inequality Reader: Contemporary and Foundational Readings in Race, Class, and Gender, édité par David B. Grusky et Szonja Szelényi. 2nd edition. Boulder: Westview Press.

Habermas, Jürgen. 1973. Öffentlichkeit. Ein Lexikonartikel. Pp. 61-69 in Jürgen Habermas. Kultur und Kritik. Verstreute Aufsätze. Frankfurt a. M.: Suhrkamp.

Habermas, Jürgen. 1978. L'espace public: archéologie de la publicité comme dimension constitutive de la société bourgeoise. Paris: Payot.

Habermas, Jürgen. 2015. Espace publique et sphère publique politique. Les racines biographiques de deux thèmes de pensée. Esprit 417: 12-25.

OIT (Organisation internationale de travail). 2014. Profits and Poverty. The Economics of Forced Labour. Genève: OIT.

Imhof, Kurt. 2011. Die Krise der Öffentlichkeit. Kommunikation und Medien als Faktoren des sozialen Wandels. Frankfurt et New York: Campus.

Imhof, Kurt. 2014. Der dünne Firnis der Zivilisation. Krisen in der Öffentlichkeit und die Krise der Öffentlichkeit. Zeitschrift für Theoretische Soziologie 3(2) : 304-338.

Krugman, Paul. 2014. Why we're in a new gilded age. The New York Review of Books 61(8). 
Lanier, Jaron. 02.07.2015. Noch erscheinen die Diktatoren des Internets milde. P. 13 in Frankfurter Allgemeine Zeitung.

Lutz, Helma. 2008. Vom Weltmarkt in den Privathaushalt. Die neuen Dienstmädchen im Zeitalter der Globalisierung. Opladen et Farmington Hills: Barbara Budrich.

Merton, Robert K. 1968. The Matthew effect. Science 159(3810) : 56-63.

Mishel, Lawrence. 2013. The CEO-to-worker compensation ratio in 2012 of 273 was far above that of the late 1990s and 14 times the ratio of 20.1 in 1965. In Economic Policy Institute, http:// www.epi.org/publication/the-ceo-to-worker-compensation-ratio-in-2012-of-273/ (29.05.2016).

Neckel, Sighard. 2010. Refeudalisierung der Ökonomie: zum Strukturwandel kapitalistischer Wirtschaft. MPIfG Working Paper 10/6. Köln : Max-Planck-Institut für Gesellschaftsforschung.

Neckel, Sighard. 2013. «Refeudalisierung» - Systematik und Aktualität eines Begriffs der Habermas'schen Gesellschaftsanalyse. Leviathan 41(1): 39-56.

Neckel, Sighard. 2014. Oligarchische Ungleichheit. Winner-take-all-Positionen in der (obersten) Oberschicht. WestEnd. Neue Zeitschrift für Sozialforschung 11: 51-63.

Neckel, Sighard. 2015. Die Ungleichheit der Märkte. Pp. 93-103 in (Un)Gerechte (Un)Gleichheiten, édité par Steffen Mau et Nadine M. Schöneck. Berlin : Suhrkamp.

Piketty, Thomas. 2013. Le capital au XXI' siècle. Paris: Seuil.

Stiglitz, Joseph A. 2012. The Prize of Inequality. New York: Norton.

Walk Free Foundation. 2014. Global Slavery Index 2014. http://www.globalslaveryindex.org/ (29.05.2016).

Wehler, Hans-Ulrich. 2013. Die neue Umverteilung. Soziale Ungleichheit in Deutschland. München: C.H. Beck. 